Revista

\title{
Multi-Ensayos
}

Vol. 7, núm. 14

ISSN: 2412-3285

https://multiensayos.unan.edu.ni

DOI: https://doi.org/10.5377/multiensayos.v7i14.12001

\section{El desarrollo de la conciencia fonológica y su influencia en el aprestamiento a la lectoescritura en los niños de 4-5 años}

\section{The development of phonological consciousness and its influence on literacy learning in children 4 to 5 years old}

\author{
Fernanda Chiriboga Posligua ${ }^{1}$ \\ Angie Huacón Pachay²
}

Recibido: 25 de abril de 2021. Aceptado: 14 de junio de 2021

\section{RESUMEN}

La conciencia fonológica ha sido un tema que ha sido abordado por pedagogos a través del tiempo, es considerada una pieza importante para el inicio del proceso de lectoescritura en los niños. La importancia de su desarrollo es que promueve en el niño un mejor proceso de enseñanza aprendizaje desde edades tempranas. En el Ecuador una de las problemáticas que se encuentran en los salones educación infantil es el prematuro comienzo del proceso de la lectura y escritura y esto se da generalmente por la presión que ejerce la familia en estos centros de educación infantil restándole importancia al aprestamiento a la lectoescritura. El presente artículo tiene el objetivo que los docentes y padres de familia tomen conciencia de la importancia de desarrollar primeramente las conciencias lingüísticas, luego continuar con el aprestamiento y por último dar inicio al proceso de la lectoescritura y de esta manera mejorar las competencias de lenguaje en los niños.

Palabras claves: Aprestamiento; conciencia fonológica; lectoescritura; cognición.

\section{ABSTRACT}

Phonological awareness has been a topic that has been addressed by pedagogues throughout time, it is considered an important piece for the beginning of the reading and writing process in children. The importance of its development is that it promotes in the child a better teaching and learning process from an early age. In Ecuador, one of the problems found in early childhood education classrooms is the premature beginning of the reading and writing process, and this is generally due to the pressure exerted by the family in these early childhood education centers, which undermines the importance of literacy training. The objective of this article is to make teachers and parents aware of the importance of first developing linguistic awareness, then to continue with the training and finally to begin the process of reading and writing and thus improve the language skills of children.

Keywords: Readiness; phonological awareness; literacy; cognition.

1 Universidad Laica Vicente Rocafuerte de Guayaquil, Ecuador. Correo electrónico: fernandachiriboga@hotmail.com. Orcid: https://orcid.org/0000-0002-0822-4485

2 Universidad Laica Vicente Rocafuerte de Guayaquil, Ecuador. Correo electrónico: angihuacon@hotmail.com (c) 2021 Revista Multi-Ensayos. 


\section{INTRODUCCIÓN}

Con el presente ensayo se pretende dar a conocer como se ha venido trabajando en el proceso de aprestamiento en los últimos años en la gran mayoría de las salas de educación infantil en el Ecuador, con la finalidad de tomar conciencia sobre la importancia del desarrollo de las conciencias lingüísticas especialmente la conciencia fonológica antes del inicio del aprestamiento, de esta manera se logrará que el proceso lectoescritura del niño sea una experiencia llena de espontaneidad y alegre, con esto se forma a seres humanos que disfrutan de una buena lectura con una capacidad crítica que les permita enfrentar los retos que la sociedad presenta.

\section{DESARROLLO}

Actualmente se han llevado a cabo diversas investigaciones sobre la conciencia fonológica ya que es considerada una pieza clave en el inicio del proceso de la lectoescritura ya que esta es necesaria para realizar una adecuada decodificación del lenguaje. Del mismo modo, se piensa que el trabajo que se realice en el aula a este respecto favorecerá el desarrollo del niño y evitará que exista fracaso escolar (Ortega, 2018). Estudios realizados han afirmado la relación del proceso de la lectoescritura y el dominio del componente fonológico y esto ha generado que se realicen diversas propuestas metodológicas para responder a los requerimientos de los docentes de educación inicial de considerar a la conciencia fonológica como principio didáctico para promover la lectoescritura (Araya, 2019).

Es necesario que en la primera infancia se haga mucho énfasis en el desarrollo de la conciencia fonológica como requisito necesario para la adquisición de la lectura solo de esta manera los infantes toman conciencia que el mundo del lenguaje está compuesto por una cantidad de sonidos que combinados forman silabas, palabras y frases que les permitirán expresar sus ideas, pensamientos y deseos. Con el desarrollo de la conciencia fonológica permitirán que los menores adquieran una mejor experiencia durante la adquisición de la lectura y escritura, y de esta manera prepararlos de una mejor manera para que pueda desenvolverse en la sociedad (Guitérrez \& Díez, 2018).

En el Ecuador se cuenta con un currículo de educación inicial (MINEDUC, 2014), que regula a las instituciones educativas del estado y las particulares, a ajustar su planificación curricular para estandarizar los objetivos de aprendizaje de esta manera desarrollar destrezas y habilidades en este ciclo infantil. Estas destrezas deben responder a las necesidades individuales que tienen los niños propios de su desarrollo evolutivo. En este documento se declaran actividades con distintos niveles de complejidad de acuerdo al nivel cognitivo del niño. Uno de los objetivos planteados en el área de comprensión y expresión del lenguaje en los niños en niños 3 a 5 años se encuentra "Comprender el significado de palabras, oraciones y frases para ejecutar acciones y producir mensajes que le permitan comunicarse con los demás" (MINEDUC, 2014 , p. 37). Por tal razón, los docentes de educación inicial deben planificar actividades que permitan desarrollar la conciencia fonológica en sus 3 fases, lo cual es imprescindible para llegar a comprender o entender las palabras para de esta manera facilitar el inicio del proceso lecto-escritor. Se ha comprobado que el trabajar la conciencia fonológica en la primera infancia permite el desarrollo de habilidades que facilitan codificación y descodificación de palabras lo que conlleva a que estos aprendan a leer en corto tiempo. 
En muchas instituciones educativas y en especial las particulares, los directivos y docentes ceden ante la presión de los representantes que desean que sus hijos adquieran conocimientos que no le corresponde a su edad cronológica, logrando acelerar el proceso de la lectura y escritura sin que estos tengan las competencias necesarias para este proceso. Esta presión que ejercen los padres subyace de la idea que los Centros de Educación Infantil (CEI) van a conocer letras y números restándole importancia al objetivo principal de aprendizaje en esta etapa que es el desarrollo de habilidades y destrezas a través del juego. El aprestamiento es un proceso necesario antes del inicio de aprender a leer y a escribir porque en esta etapa que los niños (5 años) adquieren destrezas y habilidades que les permitirán el ingreso a la Educación Básica (EGB), es importante que exista una correcta preparación antes del ingreso a la escolaridad y de esta manera lograr los éxitos esperados en relación con su desempeño académico. ¿Pero qué pasa antes del aprestamiento? ¿Los centros de Educación Infantil (CEI) preparan al niño para este proceso?, son muchas interrogantes que saltan al pensamiento por tal razón es necesario ahondar en esta problemática y reflexionar sobre la praxis diaria en Los CEI y como preparan a los niños de 3-4 años para el proceso del aprestamiento.

Por lo expuesto anteriormente es necesario que los docentes de educación tomen conciencia de cada uno de los procesos por lo que el niño debe pasar esta etapa y que estos tienen una secuencia que se debe respetar no es conveniente empezar un aprestamiento sin que estos tengan las competencias necesarias para finalizar con éxito este proceso. La gran mayoría de los CEl se observa que la adquisición de destrezas, habilidades y competencias sobre todo los procesos de aprestamiento a la lectoescritura, no son respetados con relación la edad cronológica de los niños, debido a que existen presiones y exigencias tanto de sus representantes como de las autoridades del plantel, para que aprendan de una manera acelerada, sin prestar atención a las características de desarrollo de cada etapa.

En el caso de los directivos, su interés radicaría en el aumento de las matrículas y una alta demanda escolar, restándole importancia a los procesos de aprendizaje de esta etapa del desarrollo humano esto se ve reflejados en las actividades diarias que planifican que solo se direccionan al conocimiento de letras y silabas de manera memorística continuando con un enfoque tradicional que se divorcia de las actualizaciones pedagógicas que se han realizado en los últimos años.

El panorama de esta situación en la actualidad es muy complejo por la pandemia por la que se atraviesa a nivel mundial toda la humanidad ha experimentado alteraciones en la vida cotidiana sin precedentes y unos de los sectores educativos más golpeado es el de la educación infantil.

\section{Relación del aprestamiento y la conciencia fonológica}

Arauz (2017) afirma que la etapa de aprestamiento es aquella donde se sientan las bases del aprendizaje que será útil para toda la vida. En este sentido, la autora afirma que los padres y maestros deben ser conscientes de que los niños necesitan comenzar la educación temprana para que se les proporcione un aprendizaje que contribuya al desarrollo y madurez de sus sentidos, así como las diferentes destrezas y habilidades que son necesarias para que tengan éxito en el futuro. 
Lema et al. (2019) analizan los puntos de vista de otros autores y explican que la importancia del aprestamiento radica en el impacto que éste causará durante el proceso lecto escritor, ya que este permite sentar las bases del pensamiento cognitivo y desarrollan habilidades para resolver problemas, el aprestamiento es considerado como el conjunto de habilidades y destrezas que se desarrollan en los niños antes de comenzar la educación formal, pero hacen énfasis que estas actividades deben tener un componente sensorial y lúdico.

Chávez \& Guamunshi (2011) explican que, durante la etapa de la educación inicial, el docente tiene la obligación de realizar una serie de actividades que les permitan adquirir y desarrollar todo tipo de habilidades: intelectuales, físicas y de socialización. Entre los estudios relacionados al aprestamiento, se describen algunas desde una perspectiva madurativa, que establece que la disposición para aprender depende de las habilidades y el nivel de madurez cognitiva del niño. En cambio, los enfoques modernos se refieren a la definición de aprestamiento escolar como multidimensional, con énfasis en la importancia de las habilidades personales y sociales y los roles de las familias y comunidades. Esto significa que, a pesar de que los niños tienen la necesidad de estar preparados para la escuela, las escuelas y, lo que es más, las comunidades, también deben estar preparados para apoyar el éxito futuro de los niños en los diversos dominios de desarrollo (Pan et al., 2019).

Bravo y Ortiz (2015) explican que los niños desde edades tempranas deben prepararse con ciertos aprendizajes de aprestamiento para afrontar la edad escolar, donde además se pone un énfasis especial en estimular las habilidades básicas encaminadas al aprendizaje escolar, como por ejemplo, la lectoescritura. Por otra parte, Chapelton (2018), afirma que los niños necesitarán tiempo antes de comprender y producir el lenguaje escrito. El autor también explica que el conocimiento sobre la forma en que los estudiantes aprenden a leer y escribir es importante para determinar el nivel de apoyo que deberán reciben, y afirma que cuando los niños escriben, hacen visible su pensamiento, y manifiesta que los niños tienen una gran variedad de canales para ello, por lo que es necesario evaluar la manera en que ven el mundo.

Con todo lo dicho anteriormente sobre los beneficios de la lecto-escritura se debe tener presente que uno de los principales retos que un niño enfrenta al iniciar el aprendizaje de la lectoescritura es la comprensión de la manera en que las letras (grafemas y los sonidos del habla (fonemas) se asocian entre sí. Para lograr esto, es necesario que el niño desarrolle sus habilidades fonológicas, las cuales facilitarán la reflexión y la capacidad de manipular de manera adecuada las sílabas, unidades intra-silábicas y fonemas, con lo que el niño comprenderá las relaciones entre la lengua oral y escrita (Guitérrez \& Díez, 2018).

Araya (2019) explica que, en la actualidad, existen ideas erróneas en cuanto al concepto de conciencia fonológica, ya que frecuentemente se asocia su aprendizaje con el llamado método alfabético para la enseñanza de la lecto-escritura inicial lo que conlleva a algunos docentes a realizar actividades que no corresponden. La conciencia fonológica es un término genérico que abarca una serie de conceptos. Según Goswami y Bryant (1990, citados en Irnanda, 2018), hay al menos tres formas de dividir una palabra en sus sonidos consecuentes: sílabas, unidades intra-silábicas y fonemas.

Neira (2020) indica que a fin de desarrollar la conciencia fonológica se necesita tomar en consideración los siguientes aspectos: 
- La conciencia auditiva. Habilidad con la que es posible hacer conciencia de aquellos sonidos que conforman el entorno al que el niño pertenece.

- La memoria auditiva. Esta área implica la habilidad para memorizar sonidos que posee el niño, la cual considera tanto su capacidad para retenerlos, como la evocación y la reproducción verbal.

- La discriminación auditiva. Esta área implica la habilidad del niño en identificar las diferencias entre los sonidos iguales y diferentes.

Las acciones que se realicen en aula de inicial para el desarrollo de la conciencia fonológica cumplirán un papel importante en el proceso de decodificación ya que este le despertará el interés por conocer más palabras, les llamará la atención todas aquellas actividades con relacionadas con el lenguaje como escuchar cuentos, aprender rimas, trabalenguas, etc. Solo con la adquisición del proceso de decodificación se logrará que el inicio del proceso de aprestamiento (5años) sea de una manera espontánea y placentera. No es suficiente que los niños tengan un adecuado desarrollo del lenguaje es necesario que el adulto proporcione todos los recursos necesarios para generar un espacio que estimule todas sus áreas.

\section{CONCLUSIONES}

Es necesario concientizar a los maestros, padres de familia y autoridades sobre la importancia de desarrollar la conciencia fonológica en los niños de educación inicial, puesto que es un requisito indispensable para la adquisición de la lectura en sus primeras fases, ya que es necesario que el niño descifre primero las palabras, y para lograr esto es necesario que previamente tome conciencia de que las palabras escritas están compuestas por letras que se transforman en sonidos.

Se debe tener presente que en esta etapa es relevante ya que los niños experimentan una característica, a la que María Montessori la llamó "mente absorbente", donde los infantes aprenden de manera natural, espontánea y alegre. Por otra parte, el apretamiento en la educación inicial es imprescindible porque incluye ejercicios preparatorios para la lectoescritura, y resulta fundamental para el inicio de la escolaridad. Si se desarrolla en primera instancia la conciencia fonológica y luego se inicia el proceso de aprestamiento se habrá formado un niño feliz con un amplio vocabulario que le permitirá expresarse con los otros y por ende querrá seguir experimentando con nuevas experiencias relacionadas al lenguaje.

\section{BIBLIOGRAFÍA}

Arauz, S. (2017). Incidencia del aprestamiento, lateralidad y lectoescritura en el desarrollo físico y cognitivo de los niños y niñas de la tercera etapa de Educación Inicial del centro escolar "Tierras Blancas", municipio Tuma-La Dalia, durante el I semestre del 2017. . (Tesis de Grado). Managua: Universidad Nacional Autónoma de Nicaragua.

Araya, J. (2019). Los Principios de la Conciencia Fonológica en el Desarrollo de la Lectoescritura Inicial. Revista de Lenguas Modernas(30), 163-181. Obtenido de https://revistas.ucr.ac.cr/index.php/rlm/ article/view/38981

Araya-, S., \& Espinoza, L. (2020). Aportes desde las neurociencias para la comprensión de los procesos de aprendizaje en los contextos educativos. Propósitos y Representaciones, 8(1), c312. doi:http:// dx.doi.org/10.20511/pyr2020.v8n1.312 
Arias, G., \& Ávila, Y. (2016). Construcción y validación de la prueba de conciencia fonológica A \& A. Revista de Investigación Universitaria, 2, 41-46.

Bravo, C., \& Ortiz, T. (2015). Incidencia del periodo de aprestamiento pre escolar en el desarrollo de habilidades linguisticas (pre escritura) en los niños $d 4$ a 6 años. Cuenca: UPS Cuenca.

Chapelton, T. (31 de 07 de 2018). Gow kids learn to write2018. Obtenido de https://www.britishcouncil.es/ blog/como-aprender-escribir-ingles-ninos

Chávez, E., \& Guamunshi, R. (2011). El aprestamiento escolar en el desarrollo de la motricidad fina y gruesa en niños y niñas del primer año de Educación General Básica de la Escuela "José Joaquín de Olmedo". Alausí: Universidad Estatal de Bolívar.

Guitérrez, R., \& Díez, A. (2018). Conciencia fonológica y desarrollo evolutivo de la escritura en las primeras edades. Educación XX1, 21(1), 395-415.

Irnanda. (2018). Conciencia fonológica y el desarrollo de la lectoescritura en el aprendizaje del inglés como tercera lengua. Título de Doctor en Filosofía. Universidad de West England, Bristol, UK. Obtenido de http://uwe-repository.worktribe.com

Lema, R., Tenezaca, R., \& Aguirre, S. (2019). El aprestamiento a la lectoescritura en la educación inicial. Conrado, 244-252. Retrieved from http://scielo.sld.cu/pdf/rc/v15n66/1990-8644-rc-15-66-244.pdf

MINEDUC. (2014). Currículo de Educación Inicial. Quito: MINEDUC. Retrieved from http://educacion.gob. ec/wp-content/uploads/downloads/2016/03/CURRICULO-DE-EDUCACION-INICIAL.pdf

Neira, H. (2020). Desarrollo de la Conciencia Fonológica en escolares de 5 a 7años en la Escuela de Educación Básica Zoila Aurora Palacios Cuenca 2020. Universidad de Cuenca, Cuenca.

Ortega, S. (2018). El rol de los predictores tempranos del aprendizaje de la lengua escrita en niños de educación inicial y primaria. IX Congreso Internacional de la Cátedra UNESCO para el Mejoramiento de la Calidad y Equidad de la Educación en América Latina, con base en la Lectura y la Escritura (págs. 1-5). Cuenca: UNESCO. 